\title{
An In Ovo Investigation of the Ultrastructural Effects of the Heavy Metals Cadmium and Chromium on Liver Tissue
}

\author{
Chantelle Venter ${ }^{1}$, Hester Magdalena Oberholzer ${ }^{1}$, Helena Taute ${ }^{1}$, Megan Jean Bester ${ }^{1}$; Christiaan \\ Frederick van der Merwe ${ }^{2}$ \\ 1 . Department of Anatomy, Faculty of Health Sciences, University of Pretoria, Pretoria, South Africa \\ 2. Laboratory for Microscopy and Microanalysis, Faculty of Natural and Agricultural Sciences, \\ University of Pretoria, Pretoria, South Africa
}

Many heavy metals are essential for human biological functions, but some heavy metals, especially at high concentrations, can have toxic, carcinogenic and/or teratogenic effects in humans. South Africa is the world's largest reserve of minerals and due to extensive mining activity, air and water pollution occurs [1,2]. Metal exposure occurs mostly through the ingestion of contaminated water; used for drinking, cooking and for agricultural purposes [3]. In this study the effect of heavy metals, cadmium (Cd) and chromium $(\mathrm{Cr})$ were evaluated due to the high risk of exposure in South Africa. In recent studies, high levels of $\mathrm{Cd}$ and $\mathrm{Cr}$ were found in rivers and dams in several provinces in South Africa [4-6]. The most vulnerable population to be affected by heavy metal exposure is pregnant women with developing embryos and young children living in rural communities. In the human body, $\mathrm{Cd}$ and $\mathrm{Cr}$ are metabolized differently, but the consequences are similar namely oxidative stress that leads to DNA damage, apoptosis and lipid peroxidation [7-9]. Thus, the purpose of this study was to evaluate the ultrastructural effects of $\mathrm{Cd}$ and $\mathrm{Cr}$ on liver tissue by using the in ovo model. Analytical techniques energy dispersive spectroscopy (EDS) and electron energy-loss spectroscopy (EELS) were used to confirm the presence of these metals in liver tissue.

Fertilized Broiler hatching eggs were obtained from a local farm in Bronkhorstspruit, Gauteng, South Africa. On the third day the eggs were exposed to $50 \mu \ell$ of $\mathrm{CdCl}_{2}$ at $0.074 \mu \mathrm{g} / \mathrm{m} \ell$ [physiological dose (PD)] or $74 \mu \mathrm{g} / \mathrm{m} \ell$ (x1000 PD) and $50 \mu \ell$ of $\mathrm{K}_{2} \mathrm{Cr}_{2} \mathrm{O}_{7}$ at $0.7 \mathrm{~g} / \mathrm{m} \ell$ (PD) or $700 \mu \mathrm{g} / \mathrm{m} \ell$ (x1000 PD) (n=15), dissolved in sterile water $[10,11]$. Control eggs $(n=15)$ were exposed to sterile water only. On day fourteen the embryos were terminated, the livers were removed and were prepared for transmission electron microscopy. Sections were viewed with a JEOL 2100F transmission electron microscope (TEM) and changes to hepatocyte, nucleus and mitochondria morphology were evaluated. To confirm the presence of intracellular Cd and Cr, EDS (JEOL 2100F TEM) and EELS (Tecnai HR F20 TEM) analysis were performed.

The hepatocytes of the control group had normal hepatocyte ultrastructural morphology. Hepatocytes of the Cd PD group were similar to the control with normal evenly dispersed nuclear chromatin, no mitochondrial damage and intact nuclear and cellular membranes. In contrast the Cd x1000 PD group (Fig. 1 A) showed major alterations in morphology with irregular chromatin condensation, damaged or absent organelles as well as ruptured cellular membranes. The hepatocytes in the Cr PD group showed no damage, but the mitochondria showed normal to mild inner matrix swelling. Likewise in the $\mathrm{Cr}$ x1000 PD group, indicated by figure $1 \mathrm{~B}$, the chromatin was evenly arranged and the cellular and nuclear membranes were intact with only slight damage to the mitochondrial membrane.

Evaluation of the presence of $\mathrm{Cd}$ and $\mathrm{Cr}$ in the hepatocytes with EDS provided questionable results. In contrast with EELS analysis, clear and reproducible electron spectral imaging (ESI) micrographs of metal distribution were obtained. EELS analysis revealed the presence of both $\mathrm{Cd}$ and $\mathrm{Cr}$ in the nuclei 
and mitochondria of the hepatocyte of the Cd x1000 PD (Fig. 1C) and Cr x1000 PD (Fig. 1 D) groups. The accumulation of these metals especially in the nucleus and mitochondria, indicates a possible binding affinity to the negatively charged backbone of DNA leading to altered gene and subsequent protein expression and subsequent embryo toxicity.

In conclusion, no observable differences between the PD groups and control were found, Cd x1000 PD was more toxic than the Cr x1000 PD groups and the EELS analysis confirmed these findings due to the accumulation of the metals in the nucleus and mitochondria of the hepatocytes [12].

References:

1. GF Nordberg, BA Fowler, M Nordberg, et al. in "Handbook of Toxicology of Metals", ed. Nordberg GF, Nogawa K, Nordberg M, et al., (Academic Press, Inc., Amsterdam) p. 4-9 (a).

2. R Hamann, Natural Resources Forum 28 (2004), p. 278-290.

3. OR Awofolu, Z Mbolekwa, V Mtshemla, et al., Water SA 31 (2005), p. 87-94.

4. OS Fatoki and R Awofolu, Water SA 29 (2003), p. 375-380.

5. PE Molokwane, KC Meli, et al., Water research 42 (2008), p. 4538-4548.

6. E Tempelhoff, Beeld (2013).

7. Bertin G and Averbeck D, Biochimie 88 (2006), p. 1549-1559.

8. SS Leonard, GK Harris and X Shi, Free Radical Biology \& Medicine 37 (2004), p. 1921-1942.

9. M Valko, CJ Rhodes, J Moncola, et al., Chem-Biol Interact 160 (2006), p. 1-40.

10. GF Nordberg, K Nogawa, M Nordberg, et al. in "Handbook of Toxicology of Metals", ed. GF

Nordberg, K Nogawa, M Nordberg, et al., (Academic Press, Amsterdam) p. 445-486 (b).

11. S Langård and M Costa. Chromium in "Handbook on the Toxicology of Metals", ed. GF Nordberg, BA Fowler, M Nordberg, et al., (Academic Press, Inc., Amsterdam) p. 487-510.

12. The authors would like to acknowledge Dr Cummings of the University of Western Cape, South Africa for his assistance with the EELS analysis.
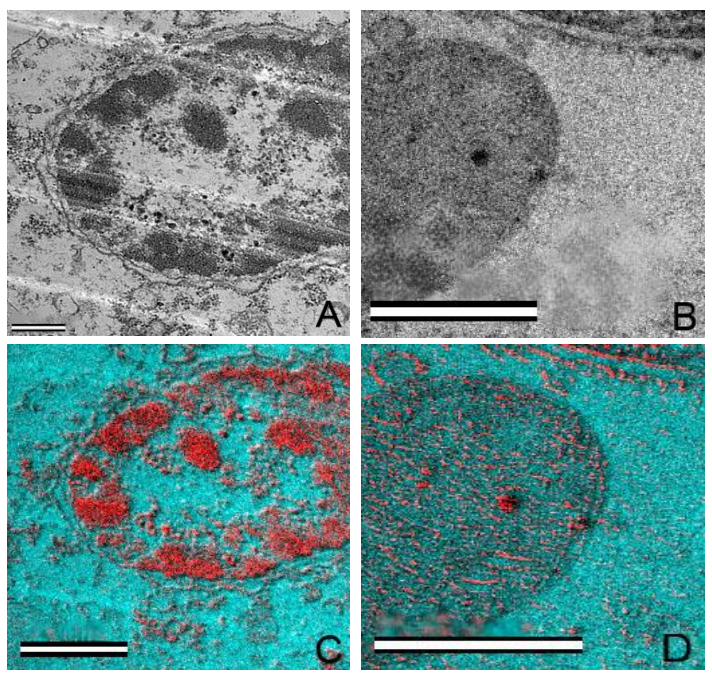

Figure 1: TEM and EELS micrographs of liver tissue exposed to Cd x1000 PD and Cr x1000 PD. Figure $\mathrm{A}$ and $\mathrm{B}$ indicate alterations to the typical morphology of the nucleus (Fig. A) and mitochondria (Fig. B) of the hepatocytes in the $\mathrm{Cd}$ x1000 PD and $\mathrm{Cr}$ x1000 PD respectively. Figure $\mathrm{C}$ is an ESI micrograph of the hepatocyte nucleus, shown in figure $\mathrm{A}$, of the $\mathrm{Cd}$ x1000 PD group with the $\mathrm{Cd}$ highlighted in red. Figure D depicts the Cr x1000 PD ESI micrograph of the mitochondria, shown in figure $\mathrm{B}$, with the $\mathrm{Cr}$ particles in red (Scale bars: A; B; D: $0.5 \mu \mathrm{m}$; $\mathrm{C}: 1 \mu \mathrm{m}$ ). 